\title{
Lessons of the month 2: FAST is not always FAST
}

\author{
Authors: Muhammad IA Qureshi ${ }^{A}$ and Ayman Osman ${ }^{B}$
}

Thrombolytic treatment with tissue plasminogen activator is an established therapy for selected patients with ischaemic stroke within a narrow time window of 3 hours from the onset of symptoms. However, severe complications and poor outcomes are likely to ensue if a patient with acute aortic dissection is inadvertently treated with thrombolytic agents. We describe a patient presenting as facial droop, arm weakness, speech disturbance and time to call emergency services (FAST) positive (clinical picture of ischaemic stroke) who received thrombolysis and in whom the underlying diagnosis of acute thoracic aortic dissection was made by a combination of chance and a high clinical suspicion.

KEYWORDS: FAST positive, thrombolysis, aortic dissection, chest pain

DOI: 10.7861/clinmed.2019-0465

\section{Case presentation}

A 65-year-old woman was admitted to emergency department as facial droop, arm weakness, speech disturbance and time to call emergency services (FAST) positive. Past medical history included hypertension and basal cell cancer. Her initial symptom was sudden onset left-sided weakness. Her computed tomography (CT) of the head was normal. She was clinically assessed by the medical team and thrombolysed for suspected stroke as per stroke on-call advice. She was also complaining of mild central chest pain which was not investigated further as initial troponins were negative and electrocardiography was normal.

Post thrombolysis, her chest pain got worse and she was reviewed by an acute physician who requested an urgent $\mathrm{CT}$ of the aorta with contrast to exclude aortic dissection. CT confirmed type A aortic dissection extending from the aortic root up to the aortic bifurcation (Fig 1). There was circumferential intimal tear with intimo-intimal glove-finger intussusception of the internal channel into the descending thoracic aorta. She was urgently transferred to the tertiary care centre under the cardiothoracic team for further management. She had an extensive surgery (aortic dissection

Authors: ${ }^{\text {A }}$ consultant physician, Worcestershire Acute Hospitals NHS Trust, Worcester, UK; ${ }^{B}$ consultant physician, Hertford County Hospital, Hertford, UK repair along with coronary artery bypass graft). Sadly, her condition did not improve postoperatively and deteriorated further and she was started on the end-of-life pathway.

\section{Discussion}

Thrombolytic treatment with tissue plasminogen activator is an established therapy for selected patients with ischaemic stroke within a narrow time window of 3 hours from the onset of symptoms. ${ }^{1}$ However, severe complications and poor outcomes

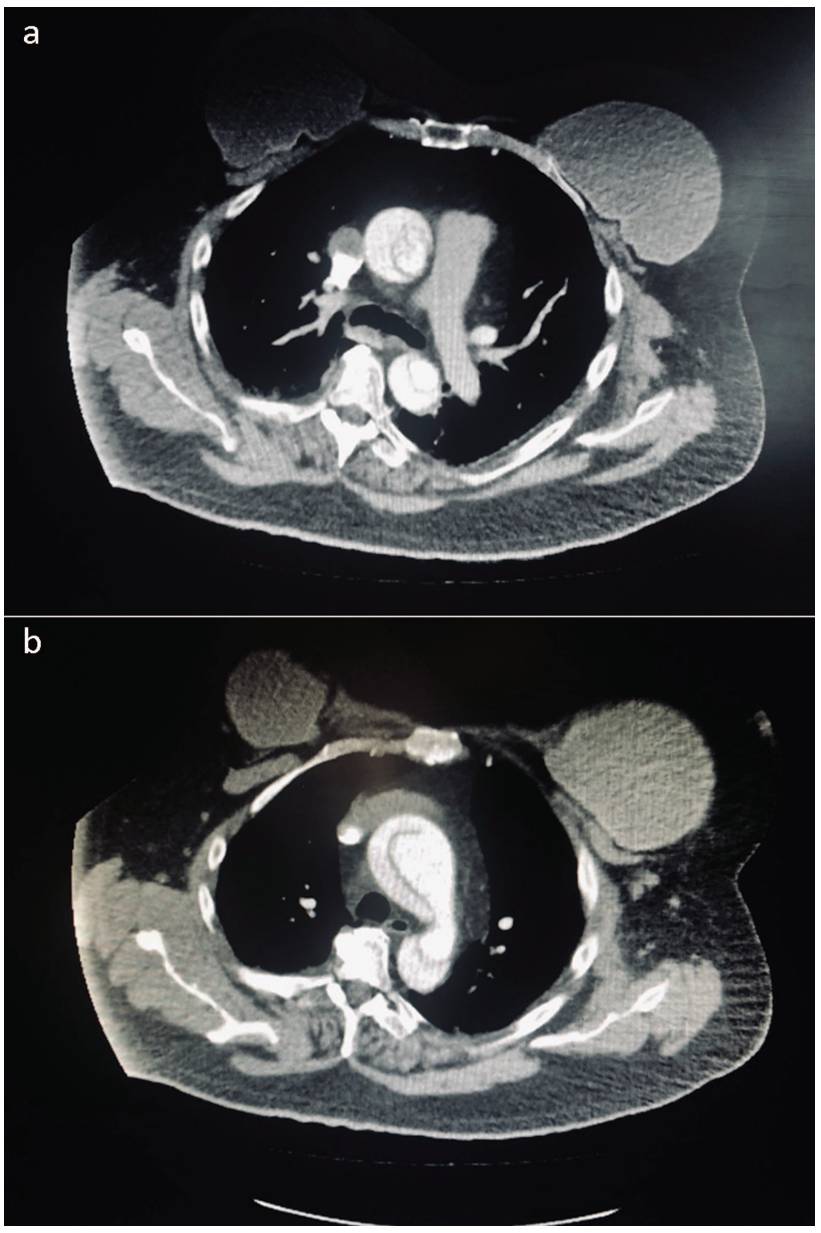

Fig 1. Computed tomography confirming type A aortic dissection extending from the aortic root up to the aortic bifurcation. 
are likely to ensue if a patient with acute aortic dissection is inadvertently treated with thrombolytic agents. ${ }^{2}$

Aortic dissection is a life-threatening, uncommon but treatable emergency that normally presents with tearing chest and back pain. It has been classified into type A (involving the ascending aorta) and type B (involving the aorta distal to the origin of the left subclavian artery).

The most essential element in the diagnosis of acute aortic dissection is a high level of clinical suspicion and the most sensitive clue is the symptom of abrupt chest or abdominal pain, the inability of the patient to describe his symptoms is highly problematic. Furthermore, pulse deficits are present in only $20-30 \%$ of cases of acute aortic dissection and may well be transient. ${ }^{3}$

Clinical suspicion may also be aroused by the detection of a widened mediastinum on plain chest X-ray, but in up to $20 \%$ of cases the chest $X$-ray may be negative. ${ }^{4}$

Management is mainly surgical in type A dissection and medical management is favourable in uncomplicated type $B$ dissection.

Acute dissection of the ascending aorta is highly lethal with a cumulative mortality rate of 1-2\% per hour early after the onset of symptoms. With medical management alone, the mortality rate peaks at nearly $20 \%$ and $50 \%$ after 24 hours and 1 month, respectively. ${ }^{5}$

Acute type A aortic dissection is a surgical emergency since these patients are at high risk for a life-threatening complication such as aortic regurgitation, cardiac tamponade, stroke, frank rupture and myocardial infarction. ${ }^{3}$

The survival benefits of surgery were illustrated in an International Registry of Acute Aortic Dissection review of 547 patients with a type A dissection. ${ }^{6}$ Eighty per cent of patients were treated surgically. The main cited reasons for medical therapy were comorbid conditions, advanced age (mean of 80 years) and patient refusal. The in-hospital mortality rates were $27 \%$ and $56 \%$ for surgical and medical therapy, respectively, which is a difference that was due, in part, to increased comorbidity in medically treated patients. Not surprisingly, the patients who died had a higher rate of in-hospital complications, including neurologic deficits, coronary or mesenteric ischaemia, acute renal failure, and limb ischaemia.

\section{Learning points}

In summary, a diagnosis of aortic dissection should always be considered in any patient presenting as FAST positive, especially when considering thrombolysis. However, it is not practically possible to consider diagnostic testing in each patient due to multiple reasons, eg comorbidities, chronic kidney disease etc. Basic clinic skills, such as taking a detailed medical history, thorough physical examination and using simple diagnostic aids may help in reaching the final diagnosis promptly. In this case, a timely diagnosis may be life-saving.

\section{Patient consent}

Sadly, the patient died and there was no imminent family available for this purpose, however, steps have been taken to anonymise her details and remaining information was regarded as clinically relevant.

\section{References}

1 The National Institute of Neurological Disorders and Stroke rt-PA Stroke Study Group. Tissue plasminogen activator for acute ischemic stroke. N Engl J Med 1995;333:1581-7.

2 Kamp TJ, Golschmidt-Clermont PJ, Brinker JA, Resar JR. Myocardial infarction, aortic dissection, and thrombolytic therapy. Am Heart J1994;128:1234-7.

3 Nienaber CA, Eagle KA. Aortic dissection: new frontiers in diagnosis and management: Part I: from etiology to diagnostic strategies. Circulation 2003;108:628-35.

4 Earnest F 4th, Muhm JR, Sheedy PF 2nd. Roentgenographic findings in thoracic aortic dissection. Mayo Clin Proc 1979;54:43-50.

5 Nienaber CA, Eagle KA. Aortic dissection: new frontiers in diagnosis and management: Part II: therapeutic management and follow-up. Circulation 2003:108:772-8.

6 Mehta RH, Suzuki T, Hagan PG et al. Predicting death in patients with acute type a aortic dissection. Circulation 2002;105:200-6.

Address for correspondence: Dr Muhammad IA Qureshi, Worcestershire Acute Hospitals NHS Trust, Charles Hastings Way, Worcester, Worcestershire WR5 1DD, UK.

Email: mia.qureshi@nhs.net 